\title{
Real-Time Vehicle License Plate Recognition System Using Adaptive Heuristic Segmentation Algorithm
}

\author{
Moon Yong $\mathrm{Jin}^{\dagger} \cdot$ Jong Bin Park ${ }^{++} \cdot$ Dong Suk Lee ${ }^{+++} \cdot$ Dong Sun Park ${ }^{+++\dagger}$
}

\begin{abstract}
The LPR(License plate recognition) system has been developed to efficient control for complex traffic environment and currently be used in many places. However, because of light, noise, background changes, environmental changes, damaged plate, it only works limited environment, so it is difficult to use in real-time. This paper presents a heuristic segmentation algorithm for robust to noise and illumination changes and introduce a real-time license plate recognition system using it. In first step, We detect the plate utilized Haar-like feature and Adaboost. This method is possible to rapid detection used integral image and cascade structure. Second step, we determine the type of license plate with adaptive histogram equalization, bilateral filtering for denoise and segment accurate character based on adaptive threshold, pixel projection and associated with the prior knowledge. The last step is character recognition that used histogram of oriented gradients (HOG) and multi-layer perceptron(MLP) for number recognition and support vector machine(SVM) for number and Korean character classifier respectively. The experimental results show license plate detection rate of 94.29\%, license plate false alarm rate of $2.94 \%$. In character segmentation method, character hit rate is $97.23 \%$ and character false alarm rate is $1.37 \%$. And in character recognition, the average character recognition rate is $98.38 \%$. Total average running time in our proposed method is $140 \mathrm{~ms}$. It is possible to be real-time system with efficiency and robustness
\end{abstract}

Keywords : License Plate Recognition(LPR), Heuristic Segmentation, AdaBoost, Histogram of Oriented Gradients(HOG)

\section{적응 휴리스틱 분할 알고리즘을 이용한 실시간 차량 번호판 인식 시스템 \\ 진 문 용 $\cdot$ 박 종 빈 $^{++} \cdot$ 이 동 석 ${ }^{+++} \cdot$ 박 동 선 ${ }^{++++}$}

\begin{abstract}
요 약
차량 번호판 인식 시스템은 복잡한 교통환경의 효율적 관리를 위해 발전되어 현재 많은 곳에 사용되고 있다. 그러나 조명, 잡음, 배경 변화, 번호판 훼손 등 환경변화에 큰 영향을 받기 때문에 제한된 환경에서만 동작하며, 실시간으로 사용하기 어렵다. 본 논문에서는 조명변 화와 잡음에 강건하며 빠른 번호판 인식을 위한 휴리스틱 분할 알고리즘 및 이를 이용한 실시간 번호판 인식 시스템을 제안한다. 첫 번째 단계는 Haar-like 특징과 Adaboost를 이용하여 번호판을 검출한다. 이 방법은 적분영상을 이용하며 케스케이드 구조로 구성되어 있어 빠른 검출이 가능하다. 두 번째 단계에서 적응 히스토그램 평활화 방법과 노이즈를 경감시키는 바이레터럴 필터를 이용하여 번호판의 종류를 결 정한 후, 번호판 종류에 따라 적분영상을 이용한 적응 이진화, 픽셀 프로젝션, 사전지식 등을 기반으로 빠르고 정확한 문자 분할을 한다. 세 번째 단계에서는 HOG와 신경망 알고리즘을 이용하여 숫자를 인식하고, SVM을 이용해 한글을 인식한다. 실험결과는 번호판검출에 $94.29 \%$ 의 검출률, $2.94 \%$ 의 오경보율을 보이며, 문자분할에서는 검출률 $97.23 \%, 2.94 \%$ 의 오경보율을 보였다. 문자인식에서 평균 인식률은 $98.38 \%$ 이다. 평균 운용시간은 $140 \mathrm{~ms}$ 으로 빠르고 강인한 실시간 시스템을 만들 수 있다.
\end{abstract}

키워드 : 번호판인식, Heuristic Segmentation, AdaBoost, Histogram of Oriented Gradients(HOG)

※ 이 논문은 미래창조과학부 및 정보통신산업진흥원의 산학협력 특성화 지원사업의 연구결과로 수행되었음(NIPA-2013-H0803-13-1003)

+ 비 회 원: 현대모비스 연구개발본부 연구원

†† 준 회 원: 전북대학교 전자정보공학부 박사과정

†+† 준 회 원 전북대학교 젖자정보공학부 석사과정

+†+† 정 회 원: 전북대학교 전자공학부 교수

Manuscript Received : February 28, 2014

First Revision : June 27, 2014

Accepted: July 28, 2014

* Corresponding Author: Dong Sun Park(dspark@ibnu.ac.kr)

\section{1. 서 론}

현재의 복잡한 교통 환경의 효율적 관리가 요구됨에 따라 지능형 교통관리 시스템 구성의 필요성이 높아졌다. 공학적 으로 지능형 교통관리는 지능형 인프라 시스템과 지능형 차 량 시스템으로 구분할 수 있다. 번호판 인식은 지능형 인프 
라 시스템의 가장 중심기술로서, 고속도로 통행료 지불 시 스템, 주차장 출입관리 시스템, 교통량 감시 및 도시 출입 관리 시스템 등 다양한 응용분야에서 인간의 단순작업을 대 행하는 중요한 역할을 수행할 수 있다. 번호판 인식(LPR : License Plate Recognition) 시스템은 번호판 검출, 문자 분 할, 문자 인식의 3 단계로 구성된다.

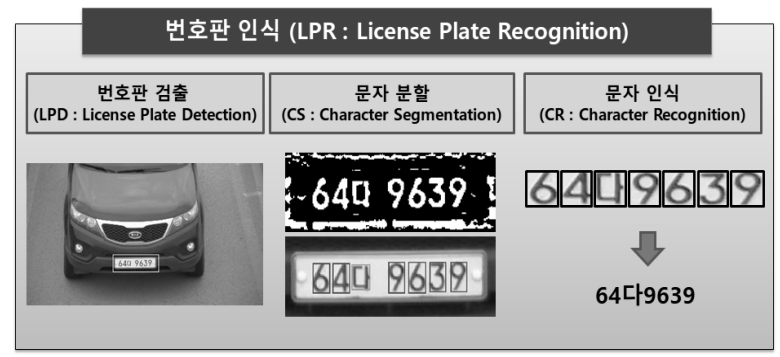

Fig. 1. System configuration of License Plate Recognition

우리나라 번호판은 녹색, 흰색, 노란색의 색상, 1 또는 2 줄의 번호판 줄 수, 두께가 다른 고딕계열 글꼴로 구성되어 있다. 이러한 환경에서 인식 알고리즘은 실시간 처리를 위 한 빠른 연산, 다양한 번호판 인식과 자연 환경, 인공 환경 의 급격한 변화에 따른 입력 데이터의 부족에 강건함이 요 구된다. 본 논문에서는 조명변화와 잡음에 강건하고 빠른 번호판 인식을 위한 문자 분할 알고리즘을 제안하고 이를 이용한 실시간 번호판 인식 시스템을 제안한다.

\begin{tabular}{|c|c|c|c|c|c|}
\hline \multirow{2}{*}{\multicolumn{2}{|c|}{$\begin{array}{l}\text { 구 분 } \\
\text { (년도) }\end{array}$}} & \multicolumn{2}{|c|}{ 소형 } & \multicolumn{2}{|c|}{ 대형(4톤 이상) } \\
\hline & & 비영업 & 영업 & 비영업 & 영업 \\
\hline $\begin{array}{c}1996 \\
\sim \\
2003\end{array}$ & 세로 & $\begin{array}{l}- \text { 서용 } 11 \cdot{ }^{\circ} \\
\text { 가1234 }\end{array}$ & & & \\
\hline $\begin{array}{c}2004 \\
\sim\end{array}$ & 세로 & 2764 & 바 3108 & & \\
\hline 2005 & & $3.55 \times 170 \mathrm{~mm}$ & $395 \times 170 \mathrm{~mm}$ & 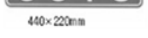 & war $20 \mathrm{man}$ \\
\hline 2006 & 가로 & 5273108 & 술 52 바 3108 & & \\
\hline 현행 & 세로 & 3942764 & 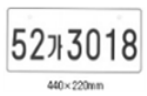 & 바31울 52. & 바 2764 \\
\hline
\end{tabular}

Fig. 2. Various Licence plates type of Korea

첫 번째 단계인 번호판 검출에서는 Haar-like 특징 및 빠 른 속도를 위해 적분영상과 Casecade 구조로 된 AdaBoost 를 사용한다. 또한 오검출 영상을 필터링하여 오경보율 (FAR : False Alarm Rate)를 줄인다.

두 번째 단계에서는 적응 히스토그램 평활화 방법과 바이 레터럴 필터를 이용하여 번호판 종류를 결정한 후, 번호판 종 류에 따른 기반 지식을 이용하여 정확한 문자 분할을 한다.

세 번째 단계에서는 HOG(Histogram of Oriented Gradients)와 신경망을 이용한 숫자 인식과, SVM(Support Vector Machine) 을 이용한 한글 인식으로 문자를 인식한다.
2절에서 기존 연구를 살펴보고, 3절에서 본 논문의 제안 알고리즘을 상술하며, 4절에서 실험을 통해 기존 방법과의 성능을 평가하고, 5 절에서 결론을 맺는다.

\section{2. 기존 연구}

번호판 인식은 단일 시스템으로 구성하기 어렵고, 연구 영역이 광범위하기 때문에 Fig. 1처럼 번호판 검출, 문자 분 할, 문자 인식의 3 단계로 나뉘어 연구되어 왔다.

\section{1 번호판 검출}

우리나라 번호판은 Fig. 2와 같은 직사각형 형태이다. 사 각형의 경계선과 엣지를 검출하면 번호판을 검출할 수 있는데, 엣지 기반 방법은 간단하고 빠르지만 복잡한 영상에서는 적용하기 어려운 단점이 있다. Kamat는 이 단점을 극복하 기 위해 허프 변환을 이용한 방법을 제안하였는데 검출률을 높인 반면, 느리고 메모리를 많이 사용하는 단점이 있다[1]. 번호판 추출 방법 중에 전역 정보를 이용한 방법도 있다. $\mathrm{CCA}$ (connected component analysis)는 이진영상에서 연결 된 픽셀들을 레이블링하여 위치와 크기 정보를 알 수 있어 모폴로지 기법을 동시에 이용하여 번호판을 검출한다[2].

\section{2 문자 분할}

Fig. 2에서 보는 바와 같이 우리나라의 번호판은 녹색, 흰 색, 노란색으로만 구성되어 있는데 Deb은 HSI 공간기반의 검출방법을 제안하였다[3]. $\mathrm{HUE}$ 값을 기반으로 후보영역을 설정한 후 번호판의 기하학적 특성과 위치 히스토그램을 이 용하여 번호판을 검출하는 방법이다. 이는 훼손되거나 틀어 진 번호판을 검출할 수 있는 장점이 있지만 조명조건과 노 이즈에 민감한 단점이 있다.

최근 연구는 기계학습을 이용하고 있다. 기계학습 방법인 AdaBoost는 2004년 얼굴검출에 관한 연구로 소개되었는데 번호판의 경우 질감특징 때문에 Haar-like 특징 기반의 AdaBoost를 이용한 연구가 많이 이루어지고 있다[4, 5, 6].

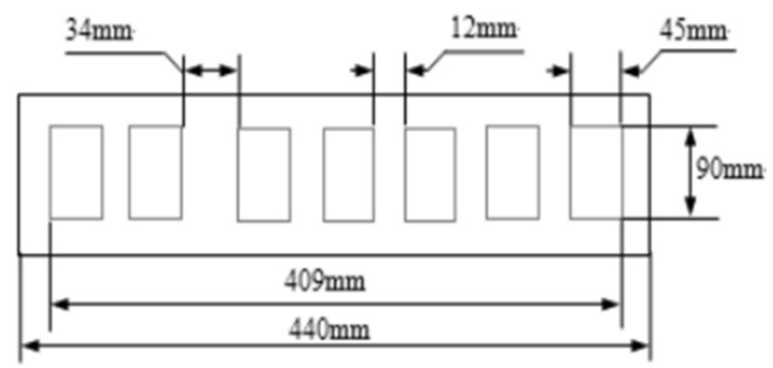

Fig. 3. Example of Character Segment using prior knowledge

번호판 영역이 추출된 후 Fig. 3과 같이 번호판 내 문자 영역이 분할되어야 한다. 검출된 번호판이 불균일한 픽셀분 포, 조명변화, 노이즈 등의 문제점을 해결하기 위해 지역적 으로 처리하는 적응 이진화 방법을 기반으로 이진영상을 구 성, 분할 연구를 한다.[7] 
픽셀 연결성을 이용한 분할 방법 $(\mathrm{CCA})$ 는 이진영상에서 연결된 픽셀들의 정보를 통해 크기, 가로세로 비율을 알 수 있다.[8] 이를 이용하여 구현이 가능하고 회전된 번호판에도 강건한 장점이 있지만 문자별 픽셀들이 붙어 있거나 연결성 이 부족한 경우 분할이 실패할 수 있다.

프로젝션은 문자분할의 고전적인 방법으로 현재에도 많이 사용된다.[9] 번호판의 이진영상에서 잡음제거 및 모폴로지 연산을 통해 전처리 후 수직방향과 수평방향으로 프로젝션 을 하면 문자들을 독립적으로 분할할 수 있지만 회전된 번 호판과 잡음이 있는 경우 성능이 낮다. 차량 번호판은 문자 들의 위치, 크기, 폰트와 문자간 거리가 정해져 있다. 이 사 전지식을 이용하여 간단하게 문자들을 분할할 수 있지만,[10] 제한적이며 변화에 민감하기 때문에 잘못된 분할 결과를 가 져올 수 있다.

\section{3 문자 인식}

문자 인식의 경우 분할된 문자들의 크기가 일정하지 않으 므로 같은 크기로 정규화 과정이 필요하며 번호판의 종류에 따라 다른 폰트를 사용하기 때문에 어려움이 있다.

문자를 인식하는 방법은 픽셀 값을 이용하는 템플릿매칭, 문자특징 추출을 통한 기계학습방법이 있다. 첫 번째 방법 은 문자 간 마할라노비스 또는 해밍 거리를 이용한 유사도 를 기준으로 문자를 인식하고 두 번째 방법은 수평 수직 프 로젝션을 통한 특징[11], 체인코드를 이용한 특징, HOG를 이용한 특징[12], SVM[13], HMM[14] 등 기계학습을 이용하 여 학습 후 인식한다.

\section{3. 제안한 번호판 인식 시스템}

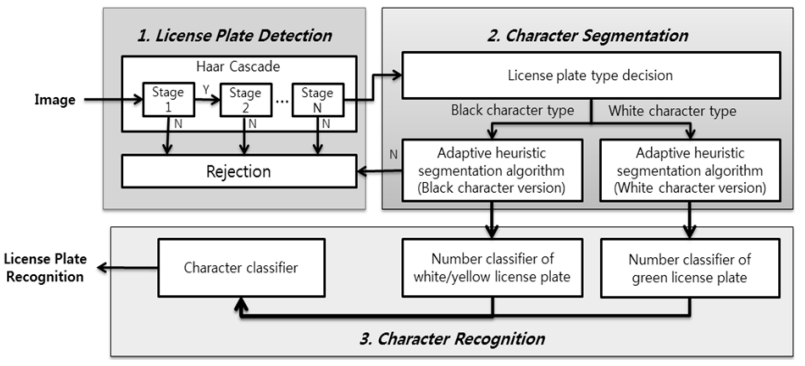

Fig. 4. The Structure of the proposed LPR

Fig. 4는 본 논문에서 제안하는 LPR구조를 보여주고 있다. 본 논문에서는 빠른 번호판 검출, 조명변화와 잡음에 강건 하고 빠른 번호판 인식을 위한 분할 알고리즘, 분할된 번호 판의 종류별 문자구분을 통한 문자 인식을 제안한다.

3.1 Haar-like feature와 AdaBoost를 이용한 번호판 검출

Papageorgiou 등은 객체의 공통 특징을 기술하기 위해 Haar wavelet을 사용할 것을 제안하였고[15], Viola와 Jones 는 이를 확장시킨 Haar-like feature의 사용을 제안하였다 [16]. Haar-like feature는 Fig. 5과 같이 두 개 이상의 인접
한 사각형 영역들로 구성되며 그 값은 영역들 간의 밝기차 로 정의된다. Fig. 5에서는 번호판의 기본 특징인 엣지 특징 과 선 특징을 보여준다.

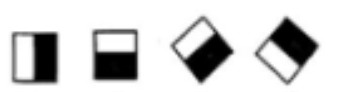

a) Edgy feature

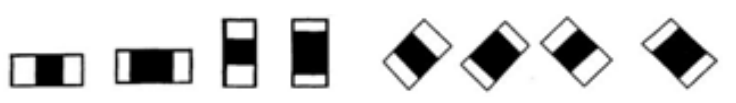

b) Line feature

Fig. 5. Haar-like feature

Haar-like feature는 적분영상을 사용하여 그 크기와 상관 없이 일정한 연산량으로 계산할 수 있다. 적분영상은 각 픽셀 이 영상의 원점으로부터 해당 픽셀 위치까지의 사각형 영역 의 모든 픽셀들의 합을 갖는 영상이다. Fig. 6에서 사각형 $\mathrm{D}$ 영역의 픽셀들의 합은 적분영상에서 4 개의 픽셀만을 참조하 여 계산할 수 있다. 적분영상에서의 픽셀 1 은 $\mathrm{A}$ 영역의 픽셀 들의 합이며 픽셀 2 는 $\mathrm{A}$ 와 $\mathrm{B}$ 영역의 합, 픽셀 3 은 $\mathrm{A}, \mathrm{C}$ 영역 의 합, 그리고 픽셀 4 는 $\mathrm{A}, \mathrm{B}, \mathrm{C}, \mathrm{D}$ 영역의 합이므로 $\mathrm{D}$ 영역 픽셀들의 합은 적분영상의 4 개 픽셀을 참조하여 $4+1-(2+3)$ 으 로 구할 수 있다[17].

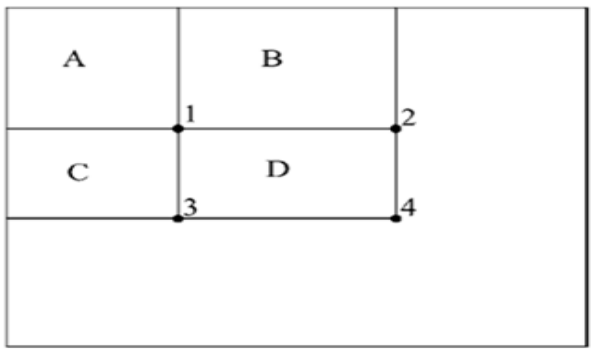

Fig. 6. Calculation of integral Image

AdaBoost는 간단한 약분류기들의 선형 결합으로 이루어 진 강분류기를 만드는 알고리즘이다.

훈련영상이 $\mathrm{n}$ 개가 있을 때 $\left(x_{1}, y_{1}\right) \cdots,\left(x_{n}, y_{n}\right)$ 의 데이터셋 으로 표현할 수 있다. 이때 $y$ 는 번호판 영상이면 0값을, 배 경 영상이면 1 값을 갖는다. 초기에는 $y_{i}$ 값을 가중치 $w_{1, i}$ 에 대해 이항분포로 설정한다. 아래 의사코드 알고리즘은 가중 치 갱신 방법을 보여준다.

for $\mathrm{t}=1, \cdots \mathrm{T}$;

$/ / \mathrm{T}$ 는 강분류기를 구성할 약분류기의 갯수

$w_{t, i} \leftarrow \frac{w_{t, i}}{\sum_{j=1}^{n} w_{t, j}} / /$ 가중치 정규화

$E_{t}=\min _{f, p, \theta} \sum_{i}\left|h\left(x_{i}, f, p, \theta\right)-y_{i}\right|$

$/ /$ 약 분류기 중 가중치 $E_{t}$ 를 최소화 하는 $f, p, \theta$ 를 정의 


$$
\begin{aligned}
& w_{t+1, j}=w_{t, i} \beta_{t}^{1-e_{i}} \\
& \quad / / \text { 영상 } x_{i} \text { 가 올바르게 분류되면 } e_{i}=0, \text { 아니면 } e_{i}=1 \\
& \beta_{t}=\frac{E_{t}}{1-E_{t}} \\
& / / \text { 최종 강분류기 } C(x) \text { 는 } \\
& C(x)= \begin{cases}1 & \sum_{t=1}^{T} \alpha_{t} h_{x}(x) \geqq \frac{1}{2} \sum_{t=1}^{T} \alpha_{t} \\
0 & \text { otherwise }\end{cases} \\
& \quad\left(\alpha_{t}=\log \frac{1}{\beta_{t}}\right), h_{x}(x) \text { 는 약분류기, }
\end{aligned}
$$

위 알고리즘을 통해 최종 강분류기를 생성하고 이 강분류 기를 케스케이드 구조로 사용함으로써 빠른 번호판 검출을 할 수 있다. 검출속도를 늘리기 위해 입력영상이나 프레임이 들어오면 미리 설정한 크기로 줄인다. 이 과정에서 슬라이딩 윈도우를 이용하여 영상 내 번호판을 검출하게 된다. Fig. 7 은 이러한 과정을 보여준다. 왼쪽에서 오른쪽으로, 위쪽에서 아래쪽으로 이동하며 검출할 ROI(Region of Interest)에 AdaBoost로 훈련된 번호판 검출기를 적용한다. 검출된 번호 판 중 오검출된 부분이 있을 수 있기 때문에 문자 분할 필 터를 이용해 이를 제거한다. 문자 분할 필터의 자세한 내용 은 다음에서 설명한다.
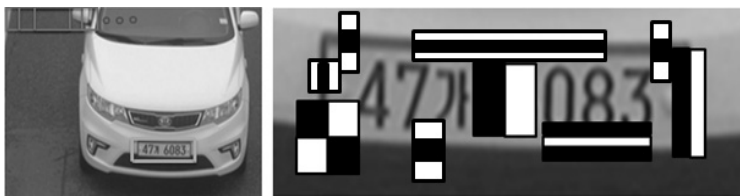

Fig. 7. Apply a sliding window and Example of LPR with trained AdaBoost

3.2 적응 휴리스틱 분할

$\mathrm{LPR}$ 의 인식 단계 성능을 극대화시키기 위해 번호판의 문 자를 개별 분할할 필요가 있다. 한국 번호판의 경우 다양한 종류와 폰트에 따라 적합한 알고리즘을 이용해야 한다. Fig. 8은 번호판 종류를 판별하는 과정을 설명한다.

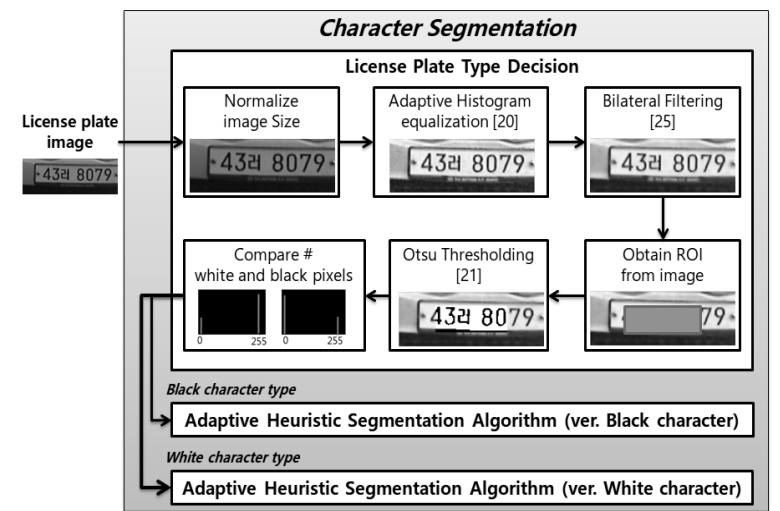

Fig. 8. Determining the type of license plate algorithm
검출된 번호판 영상은 크기 정규화 처리를 한 후에 적응 히스토그램 평활화 방법을 적용한다[18]. 적용구역을 지역 적으로 처리하면 평활화 효과가 극대화되어 영상의 전체적 contrast가 높아진다. 검출된 번호판 영상에서 픽셀들의 분포 가 한쪽으로 치우쳐 불균형한 경우가 많은데 이 경우에도 적 응 히스토그램 평활화 방법을 이용하면 큰 효과를 얻을 수 있다. 다만 약간의 잡음 증가가 있을 수 있어 이를 제거하기 위해 바이레터럴 필터링(bilateral filtering)을 하였다[19].

검출된 번호판 영상에서 번호판은 중간부분에 위치해 있 으며 문자 영역이 기타 영역보다 더 넓다. 영상을 Otsu 이 진화를 적용하여 흰색, 노란색 번호판은 바탕영역이 255값, 문자영역 0 값으로 처리하고 녹색 번호판의 경우 반대로 처 리하여 번호판 색상 종류별로 문자 영역을 처리할 수 있게 하였다[20]. 만들어진 이진영상은 레이블된 블롭들의 크기, 비율 등 사전지식을 이용해 필터링한다.

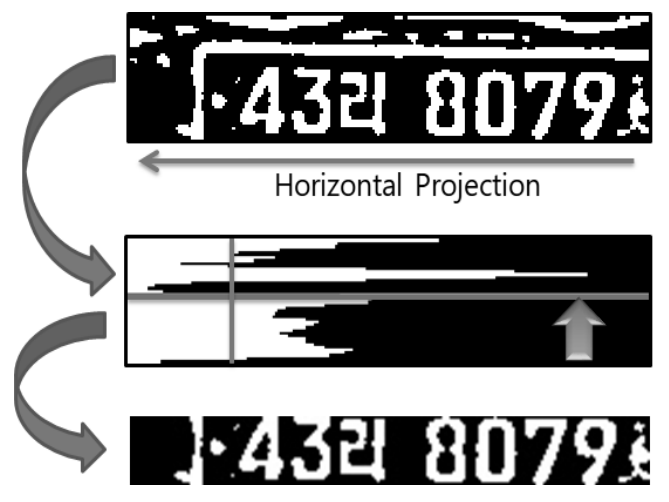

Fig. 9. Horizontal projection algorithm

Fig. 9와 같이 남아있는 픽셀들을 수평방향으로 프로젝션 하고 아래쪽에서 위쪽으로 검색한다. 여기에서 픽셀분포의 평균을 이용하여 기울기가 가장 큰 지점을 찾아 문자가 있 는 부분을 얻어 내고 추가적으로 적응 이진화를 하여 잡음 을 없앤다.

다음 단계는 사전지식을 기반으로 한 RL(Right Left), LR(Left Right) 알고리즘이다. 이 과정은 레이블된 문자들의 거리와 위치관계를 이용하여 잘못 레이블된 블롭들을 제거하는 최 종과정이다. Fig. 10의 번호판 문자들을 보면 왼쪽에 숫자 2

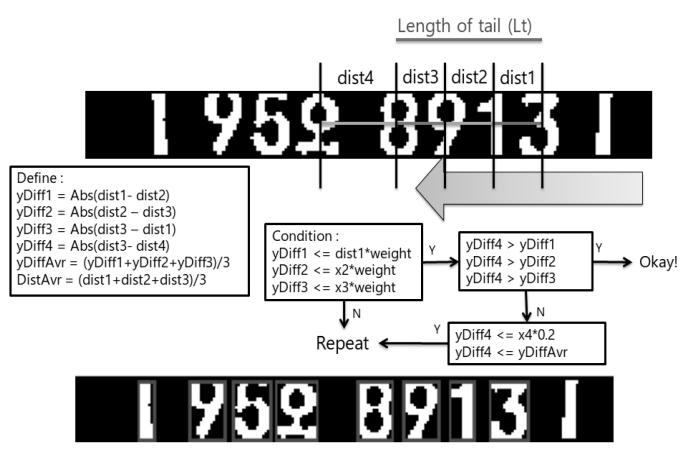

Fig. 10. RL algorithms based on prior knowledge of the application process and results 
개와 문자 1 개가 있고 오른쪽에 숫자 4 개가 있으며 이들이 서로 떨어져 있는 것을 볼 수 있다. RL 알고리즘의 경우 오 른쪽부터 왼쪽으로 5 개 블롭들의 거리관계를 살펴보고 조건 에 맞을 때까지 왼쪽으로 이동하며 체크하여 오른쪽에 있는 숫자 4 개를 결정한다.

$\mathrm{LR}$ 알고리즘도 $\mathrm{RL}$ 알고리즘과 마찬가지로 사전지식을 이용한다. 전 단계인 $\mathrm{LR}$ 알고리즘을 적용하며 얻은 4 개의 문자 중 첫 번째와 네 번째 문자의 거리를 이용하여 탐색범 위를 정한 후 왼쪽에서 오른쪽으로 이동하며 블롭관계를 체 크하여 왼쪽에 있는 숫자 2 개와 문자 1 개를 결정한다.

여기에서 조건이 만족하지 않는다면 두 줄로 이루어진 번 호판(예 : 녹색 번호판)이라 간주하고 적합한 문자 분할 알 고리즘을 적용한다.

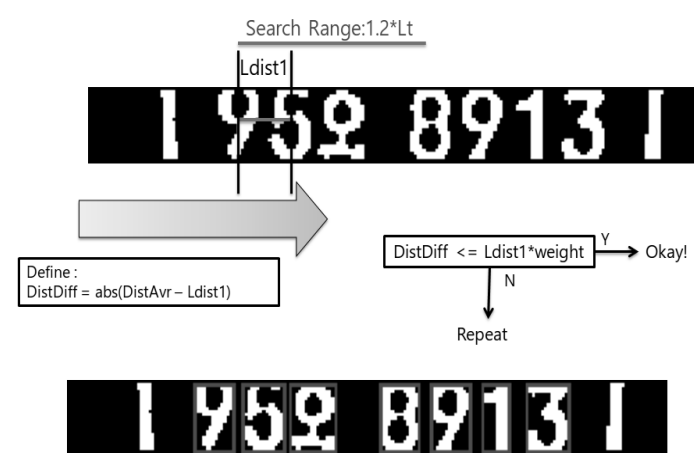

Fig. 11. LR algorithms based on prior knowledge of the application process and results

문자 중 '고, 노, 도, 로, 오' 등은 붙어 있지만 '가, 나, 다, 라' 등의 단어는 떨어져 있다. 이러한 부분을 해결하기 위해 두 번째와 세 번째 숫자 사이의 부분을 지역적으로 모폴로 지 처리를 하여 하나의 덩어리로 만들어 레이블링 한다.

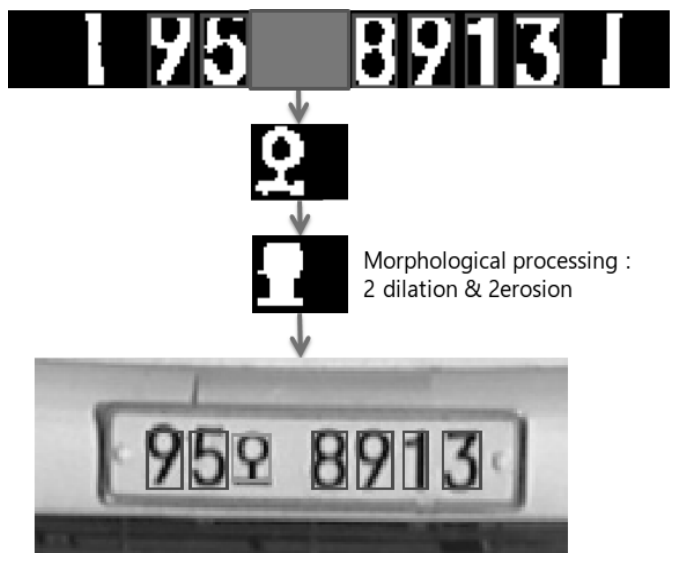

Fig. 12. Based on prior knowledge of the character for label processing area morphology

Fig. 13은 흰색 문자 분할 알고리즘의 블록 다이어그램이다. 이 경우는 녹색 번호판과 같이 두 줄로 이루어져 있는 경우 를 처리한다.

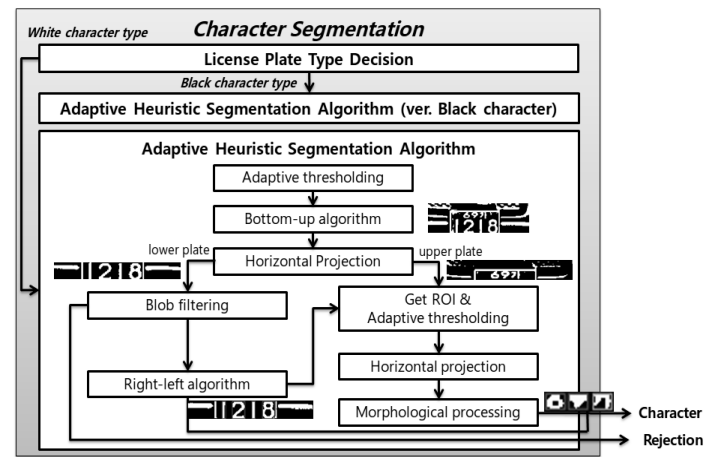

Fig. 13. Adaptive heuristic segmentation algorithm (for white character)

먼저, 앞에서 소개했던 지역 적응 이진화와 Fig. 9의 수평 적 프로젝션 방법을 사용하여 번호판의 윗부분과 아랫부분 으로 나눈다. 아랫부분을 앞에서 사용했던 $\mathrm{RL}$ 알고리즘을 사용하여 4 개의 숫자를 얻어내고 가장 왼쪽에 문자가 있는 지 결정한다. 이를 기반으로 사전지식을 활용하여 윗부분에 위치한 숫자와 문자의 $\mathrm{ROI}$ 를 얻어낸다. $\mathrm{ROI}$ 에 다시 수평적 프로젝션을 적용하여 영역을 줄이고 모폴로지 연산을 통해 레이블링하여 숫자와 문자들의 위치를 얻는다.

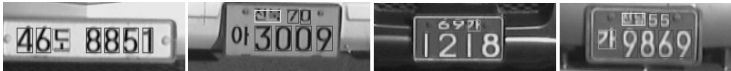

Fig. 14. Example of divide results using adaptive heuristic segmentation algorithm

\subsection{HOG와 신경망을 이용한 문자 인식}

앞 단계에서 추출된 분할 결과를 문자로 인식한다. 본 논 문에서는 번호판 색상별 폰트 차이로 흰색과 검은색의 2개 문자를 구분하여 인식한다.

문자인식에는 $\mathrm{HOG}$ 를 이용하였다. $\mathrm{HOG}$ 는 한 픽셀에 대해 $x$ 축으로 $[-1,0,1], y$ 축으로 $[-1,0,1]^{T}$ 의 마스크 필터를 이 용해 인접셀의 픽셀차이 값을 구한다. 이를 이용하여 아래 식과 같이 Magnitude와 Orientation을 구하고 Orientation의 bin에 따라 Magnitude 값을 누적시킨 히스토그램을 구할 수 있다.

$$
\begin{aligned}
& \nabla i=\left\{\begin{array}{l}
d x \\
d y
\end{array}, \text { Magnitude }=\sqrt{d x^{2}+d y^{2}},\right. \\
& \text { Orientation }=\operatorname{atan} \frac{d x}{d y}
\end{aligned}
$$

이것을 이용하여 숫자와 한글을 인식한다. 숫자의 경우 신 경망의 일종인 다층 퍼셉트론(MLP : Multi-Layer Perceptron) 을 이용해 학습하였고, 한글의 경우 37 개의 한글을 각각 선 형 SVM으로 학습하였다.

\section{4. 실험 및 성능평가}

성능평가를 위한 데이터는 Fig. 15 환경의 CMOS 카메라 에서 획득한 총 5,300 장의 $1280 \times 720$ 이미지를 사용하였다. 
이미지는 날씨 환경, 조명 환경, 밤 낮 등 다양한 환경에서 획득한 영상들이다.

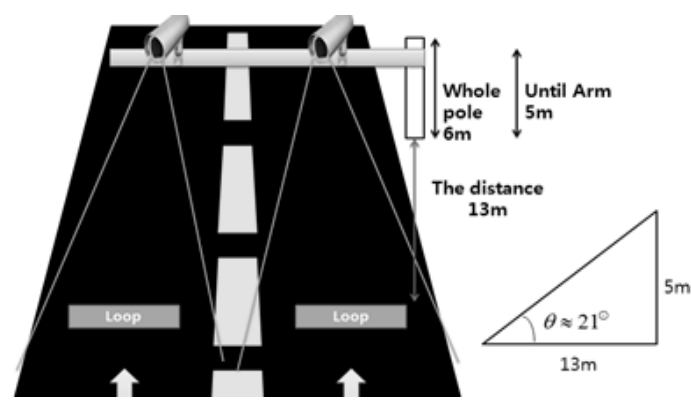

Fig. 15. Environment of evaluation data obtaining

번호판의 색상종류는 흰색 번호판 2가지, 노란색 번호판 2 가지, 녹색 번호판 2가지의 총 6 가지 종류로 한정하였다.

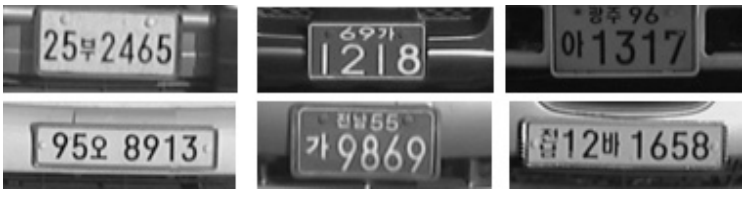

Fig. 16. Plate type used in the experiment

첫 번째 성능평가는 실제 번호판 중 검출기를 통해 번호 판을 검출한 비율인 검출률, 검출기를 통해 검출된 모든 개 수와 잘못 검출된 개수의 비율을 나타낸 오경보율, 그리고 평균 연산시간의 3 가지이다.

Table 1. The performance compare of the proposed method compared with traditional methods

\begin{tabular}{c|c|c|c}
\hline Method & $\begin{array}{c}\text { Character hit } \\
\text { rate }\end{array}$ & $\begin{array}{c}\text { False alarm } \\
\text { rate }\end{array}$ & $\begin{array}{c}\text { Average running } \\
\text { time }\end{array}$ \\
\hline $\begin{array}{c}\text { AdaBoost }+ \\
\text { CCA[5] }\end{array}$ & 0.9714 & 0.7244 & $\begin{array}{c}93.62 \mathrm{~ms} \\
\text { (Standard deviation: } \\
7.85)\end{array}$ \\
\hline $\begin{array}{c}\text { Proposed } \\
\text { (AdaBoost }+\end{array}$ & 0.9429 & 0.0294 & $\begin{array}{c}131.64 \mathrm{~ms} \\
\text { Standard deviation: } \\
50.60)\end{array}$ \\
\hline
\end{tabular}

두 번째 성능평가는 제안한 번호판 종류에 따른 전역 히 스토그램 평활화와 지역적 적응 히스토그램 평활화를 비교 하였다. 적응 히스토그램 평활화가 조명 및 잡음에 강건하 여 일반 히스토그램방법보다 $4.54 \%$ 높게 나타났다.

Table 2. Compared to normal histogram and adaptive histogram (bilateral filter $=5$ )

\begin{tabular}{c|c|c}
\hline Method & $\begin{array}{c}\text { Normal histogram } \\
\text { (Global) }\end{array}$ & $\begin{array}{c}\text { Adaptive histogram } \\
\text { (Local) }\end{array}$ \\
\hline Black Character type & $0.9620(3621 / 3764)$ & $0.9965(3751 / 3764)$ \\
\hline White Character type & $0.9173(1131 / 1233)$ & $0.9960(1228 / 1233)$ \\
\hline Total & $0.9510(4752 / 4997)$ & $0.9964(4979 / 4997)$ \\
\hline
\end{tabular}

세 번째 성능평가는 기존의 문자분할[21]과 제안한 방법 의 성능을 평가하였다.

Table 3. The performance compare of the existing character segmentation research and proposed method

\begin{tabular}{c|c|c|c}
\hline Method & Hit rate & False alarm rate & Average running time \\
\hline CCA [9] & 0.8776 & 0.1586 & $\begin{array}{c}12.50 \mathrm{~ms} \\
\text { (Standard deviation: 1.54) }\end{array}$ \\
\hline Projection [10] & 0.9114 & 0.1873 & $\begin{array}{c}13.33 \mathrm{~ms} \\
\text { (Standard deviation: 1.62) }\end{array}$ \\
\hline $\begin{array}{c}\text { Binary threshold } \\
\text { combinations [22] }\end{array}$ & 0.9452 & 0.2278 & $\begin{array}{c}58.13 \mathrm{~ms} \\
\text { (Standard deviation: 8.87) }\end{array}$ \\
\hline Proposed & 0.9723 & 0.0137 & $\begin{array}{c}24.24 \mathrm{~ms} \\
\text { (Standard deviation: 8.16) }\end{array}$ \\
\hline
\end{tabular}

Table 3은 알고리즘에서 제안한 계수 설정방법을 통해 얻은 값을 이용하여 기존 연구와 비교하였다. 분할된 문자 를 기준으로 측정한 검출률은 평균 연산시간이 $24.24 \mathrm{~ms}$ 이며 $27.23 \%$ 의 성능을 보이고 $1.37 \%$ 의 오경보율을 보여 기존 방 법보다 빠르고 높은 성능을 나타냈다.

Fig. 17은 제안한 적응 휴리스틱 분할 알고리즘이 번호판 종류에 상관없이 분할됨을 보여준다. 숫자는 빨간색, 한글은 녹색, 지역은 노란색 사각형으로 표시하였다.

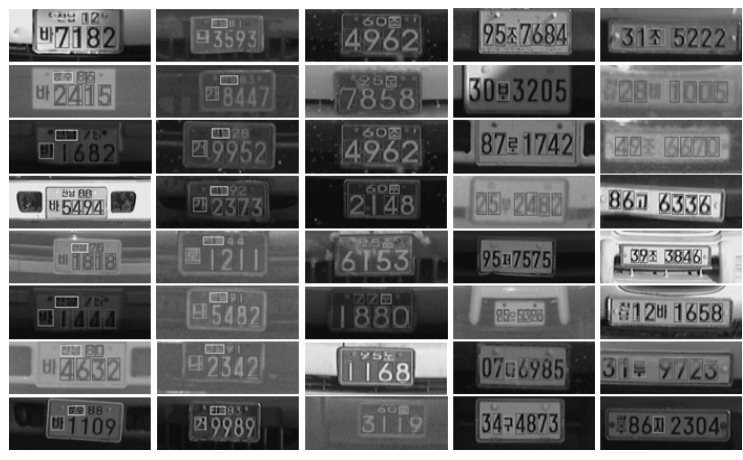

Fig. 17. Example results of applying the proposed algorithm

실험에서 얻은 문자 인식 성능을 Table 4에 표시하였다. 검은색 문자와 흰색 문자에 따라 숫자와 한글을 인식하고 보다 정확한 측정을 위해 데이터를 $20 \%$ 씩 교차 검증하였다. 검은색 문자 종류인 번호판에서 얻어진 숫자 인식률은 $99.55 \%$, 흰색 번호판의 숫자인식은 $97.84 \%$, 한글인식은 97.94\%의 문자 인식률을 보였다.

Table 4. Average number recognition using HOG and MLP (Cross-validation)

\begin{tabular}{c|c|c|c|c|c|c}
\hline Fold & 1 & 2 & 3 & 4 & 5 & $\begin{array}{c}\text { Aver } \\
\text { age }\end{array}$ \\
\hline Black Character type & 0.9972 & 0.9888 & 0.9976 & 0.9964 & 0.9976 & 0.9955 \\
\hline White Character type & 0.9714 & 0.9754 & 0.9783 & 0.9827 & 0.9841 & 0.9784 \\
\hline Korean Character & 0.9815 & 0.9756 & 0.9820 & 0.9718 & 0.9763 & 0.9774 \\
\hline Total & 0.9834 & 0.9799 & 0.9860 & 0.9836 & 0.9860 & 0.9838 \\
\hline
\end{tabular}


Fig. 18은 본 논문의 실제 차량 번호판의 문자 분할 및 인식 시스템을 구동시키는 프로그램이다. 구동 환경은 현재 일반 $\mathrm{PC}$ 에서 실시하였으나 차후 스마트폰, 테블릿 $\mathrm{PC}$ 등 이 동통신 모바일 단말기로 포함한 모든 컴퓨팅(Computing) 기 기에서 해석 가능할 것이다.
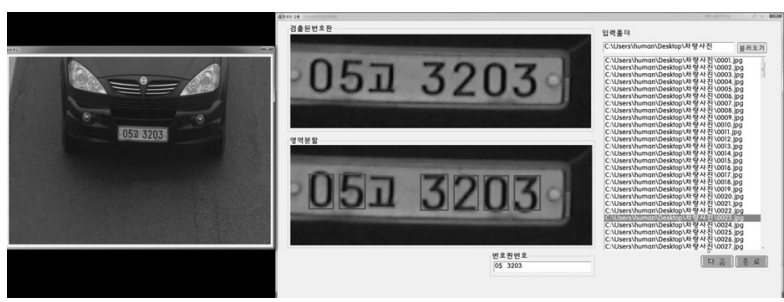

Fig. 18. Actual operating program

\section{5. 결 론}

본 논문에서는 적응 휴리스틱 분할 알고리즘을 제안하고 이 를 이용하여 실시간 LPRS(License Plate Recognition System : 차량 번호판 인식 시스템)을 제안하였다. 제안하는 LPRS는 크게 AdaBoost를 이용한 차량 번호판 검출, 적응 휴리스틱 분할, $\mathrm{HOG}$ 를 이용한 문자 인식으로 나뉜다. 번호판 검출에 서는 번호판을 잘 나타내기 위해 경계패딩처리를 한 훈련영 상을 이용하여 AdaBoost로 훈련하였다. 이 과정에서 빠른 특징계산을 위한 적분영상을 이용하였고 검출속도를 향상시 키기 위해 케스케이드 구조로 구성하였다. 문자 분할에서는 조명변화에 강건한 적응 평활화와 바이레터럴 필터를 이용 하여 번호판의 종류를 먼저 분류하고 분류된 번호판에 따라 적응 이진화, 적분영상, $\mathrm{CCA}$,프로젝션, 사전지식 등 다양한 방법들을 결합하여 문자분할의 성능을 높였다. 제안한 방법 의 문자 검출률은 $97.23 \%$, 오경보율은 $1.37 \%$, 평균 연산시 간은 $24.24 \mathrm{~ms}$ 로 기존방법들과 비교하여 빠르고 높은 성능을 보였다. 또한, 문자 분할과정 중 잘못 검출된 번호판을 제거 하여 AdaBoost만 이용하여 번호판을 검출하였을 경우와 비 교해 오경보율을 $69.5 \%$ 낮추고 $94.29 \%$ 의 검출률을 보였다. 문자 인식단계에서는 조명과 잡음에 강건한 $\mathrm{HOG}$ 특징을 이 용하였다. 숫자 인식은 검은색과 흰색 숫자 종류에 따라 신 경망을 구성하였고 한글 인식은 37개의 선형 SVM을 이용 하여 다중 분류기를 구성하였다. 숫자와 한글을 포함한 총 문자 인식결과는 평균 $98.38 \%$ 의 인식률을 보였다.

제안한 방법은 다양하고 복잡한 환경에서 강인한 성능을 갖고 번호판 검출부터 문자인식까지 약 $140 \mathrm{~ms}$ 의 짧은 시간 이 소요되기 때문에 실시간 번호판 인식을 필요로 하는 많 은 응용에 쓰일 것으로 예상된다.

그러나 전체 과정을 비교하였을 때 번호판 검출과정에서 많은 시간이 소요되었다. 향후 이를 줄이기 위해 눈에 띄는 부분을 나타내는 세일런스 맵을 이용하여 ROI를 설정한 후 AdaBoost를 적용하는 연구를 진행할 것이다[22].

\section{References}

[1] V. Kamat and S. Ganesan, "An efficient implementation of the Hough transform for detecting vehicle license plates using DSPs," Proc. Real-Time Tech Applicat. Symp., pp.58-59, 1995.

[2] P. Wu, H.-H. Chen, R.-J. Wu, and D.-F. Shen, "License plate extraction in low resolution video," Proc. Int. Conf. Pattern Recognit., Vol.1, pp.824-827, 2006.

[3] K. Deb and J. Kang-Hyun, "HSI color based vehicle license plate detection," Control, Automation and Systems, ICCAS 2008, International Conference on, pp.687-691, 2008.

[4] Md. M. K. Sarker, "License Plate Detection Using Cascade AdaBoost and Heuristic Energy Map," Master's degree thesis, Chonbuk National University, 2013.

[5] L. Dlagnekov, "License Plate Detection Using AdaBoost," San Diego, CA: Computer Science and Engineering Dept., 2004.

[6] S. Z. Wang and H. J. Lee, "A cascade framework for a real-time statistical plate recognition system," IEEE Trans. Inform. Forensics Security, Vol.2, No.2, pp.267-282, Jun., 2007.

[7] F. Shafait, D. Keysers, and T. M. Breuel, "Efficient Implementation of Local Adaptive Thresholding Techniques Using Integral Images," SPIE, Document Recognition and Retrieval XV, Jan., 2008.

[8] B.-F. Wu, S.-P. Lin, and C.-C. Chiu, "Extracting characters from real vehicle license plates out-of-doors," IET Comput. Vision, Vol.1, No.1, pp.2-10, 2007.

[9] T. D. Duan, T. L. H. Du, T. V. Phuoc, and N. V. Hoang, "Building an automatic vehicle license-plate recognition system," Proc. Int. Conf. Comput. Sci. RIVF, pp.59-63, 2005.

[10] Q. Gao, X. Wang, and G. Xie, "License plate recognition based on prior knowledge," Proc. IEEE Int. Conf. Automat. Logistics, pp.2964-2968, Aug., 2007.

[11] C. A. Rahman, W. Badawy, and A. Radmanesh, "A real time vehicle's license plate recognition system," Proc. IEEE Conf. Adv. Video Signal Based Surveillance, pp.163-166, Jul., 2003.

[12] I. S. Choi, M. Y. Jin, D. S. Park, "Vechicle License Plate Detection and Optical Number Recogniton Using Morphological processing and Neural Network", Proc. KISM Spring Conference, Vol.2, No.1, pp.58-61, May, 2013.

[13] K. K. Kim, K. I. Kim, J. B. Kim, and H. J. Kim, "Learning-based approach for license plate recognition," Proc. IEEE Signal Process. Soc. Workshop Neur. Netw. Signal Process., Vol.2, pp.614-623, Dec., 2000.

[14] D. Llorens, A. Marzal, V. Palazon, and J. M. Vilar, "Car license plates extraction and recognition based on connected components analysis and HMM decoding," Lecture Notes Comput. Sci., Vol.3522, pp.571-578, 2005. 
[15] V. Kamat and S. Ganesan, "An efficient implementation of the Hough transform for detecting vehicle license plates using DSPs," Proc. Real-Time Tech Applicat. Symp., pp.58-59, 1995

[16] Shan Du, Mahmoud Ibrahim, Mohamed Shehata and Wael Badawy, "Automatic License Plate Recognition (ALPR): A State-of-the-Art Review," IEEE Transactions on Circuits and Systems for Video Technology, Vol.23, No.2, pp.311-325, Feb., 2013.

[17] Shan Du, Mahmoud Ibrahim, Mohamed Shehata, and Wael Badawy, "Automatic License Plate Recognition (ALPR) : A State-of-the-Art Review," IEEE Transactions on Circuits and Systems for Video Technology, Vol.23, No.2, pp.311-325, Feb., 2013.

[18] Zuiderveld, Karel, "Contrast Limited Adaptive Histograph Equalization," Graphic Gems IV. San Diego: Academic Press Professional, pp.474-485, 1994.

[19] C. Tomasi, R. Manduchi, "Bilateral Filtering for Gray and Color Images," Proc. Int. Conf. Computer Vision (ICCV), 1998.

[20] N. Otsu, "A threshold selection method from gray-level histograms," IEEE Trans. Systems, Man, and Cybernetics Vol.9, No.1, pp.62-66, 1979.

[21] Y. Yoon, K. Ban, H. Yoon, J. Lee, and J. Kim, "Best Combination of Binarization Methods for License Plate Character Segmentation," ETRI Journal, Vol.35, No.3, pp.491-500, Jun., 2013.

[22] R. Achanta, S. Hemami, F. Estrada, and S. Susstrunk, "Frequency-tuned salient region detection," IEEE Conference on Computer Vision and Pattern Recognition, 2009.

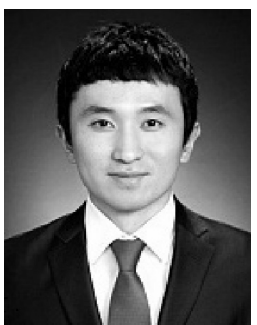

진 문 용

e-mail : qwqw2346@naver.com

2012년 전북대학교 전자공학부(학사)

2014년 전북대학교 전자정보공학부(석사)

2014년 현 재 현대모비스 연구개발본부 연구원

관심분야: 신호처리, 컴퓨터비전, 패턴인식

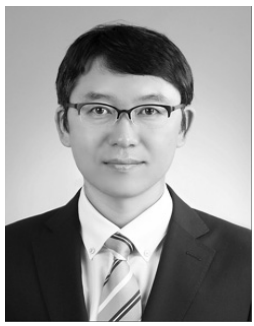

\section{박 종 빈}

e-mail : nicedugy@gmail.com

2003년 전북대학교 전자정보공학부(학사) 2005년 전북대학교 전자정보공학부(석사) 2006년 현 재 전북대학교 전자정보공학부 박사과정

관심분야: 신경망, 인공지능, 영상처리

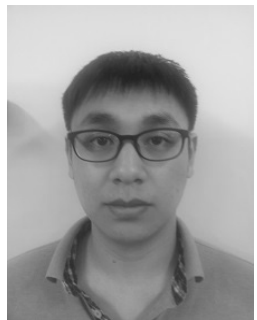

\section{이 동 석}

e-mail :krustmin@gmail.com

2014년 전북대학교 전자정보공학부(석사) 2014년 현 재 전북대학교 전자정보공학부 석사과정 관심분야 : 인공지능, 패턴인식

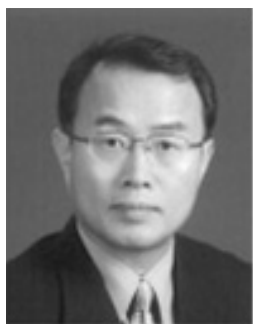

e-mail:dspark@jbnu.ac.kr 1979년 고려대학교 전기전자공학과(학사) 1984년 Missouri-Columbia(공학석사) 1991년 Missouri-Columbia(공학박사) 1991년 현 재 전북대학교 전자공학부 교수 관심분야: 신경망, 패턴인식, 영상처리, 디 지털 시스템 설계 